\title{
ABLACIÓN EXITOSA DE VÍA ANÓMALA EN PACIENTE CON ANOMALÍA DE EBSTEIN
}

\author{
SUCCESSFUL ABLATION OF ANOMALY PATHWAY IN PATIENT \\ WITH EBSTEIN ANOMALY
}

\section{MARÍA F. FIRPO' ${ }^{1}$, MARÍA FERNANDA GODOY' ${ }^{1}$ DANIELA FERNANDA CHILABERT'1, JUAN M. DOMÍNGUEZ², CAMILA ANTONIETTA ${ }^{3}$, MARCELO LANZOTTI' ${ }^{4}$, SILVANO DIANGELO ${ }^{5}$}

\section{RESUMEN}

La anomalía de Ebstein (AE) consiste en el desplazamiento congénito de las valvas septal y posterior de la tricúspide hacia el ventrículo derecho. Gracias a esto, existe dilatación auricular por "atrialización" ventricular, insuficiencia tricuspídea y falla ventricular derecha. Un 30\% se asocia con arritmias supraventriculares y síndrome de Wolff-Parkinson-White (WPW). Se presenta el caso de un paciente con ablación exitosa de vía anómala con coexistencia de AE y síndrome de WPW.

Palabras clave: anomalía de Ebstein. síndrome de Wolf-Parkinson-White.

\begin{abstract}
Ebstein Anomaly (EA) consists of the congenital displacement of the septal and posterior leaflets of the tricuspid valve towards the right ventricle. Thanks to this, there is atrial dilation due to ventricular "atrialization", tricuspid valve regurgitation and right ventricular failure. $30 \%$ is associated with supraventricular arrhythmias and Wolff-Parkinson-White syndrome (WPW). We present the case of a patient with successful ablation of abnormal pathway with coexistence of EA and WPW syndrome.
\end{abstract}

Keywords: Ebstein anomaly, Wolff-Parkinson-White syndrome.

REVISTA CONAREC 2018;33(145):188-190 | DOI:10.32407/RCON/2018145/0188-0190

\section{INTRODUCCIÓN}

La anomalía de Ebstein (AE) engloba un amplio espectro de anomalías caracterizado por diferentes grados de desplazamiento y adherencia de la valva septal y posterior de la tricúspide hacia la cavidad del ventrículo derecho, con desplazamiento y dilatación del anillo. Parte del ventrículo se introduce en la aurícula derecha, fenómeno conocido como "atrialización", con afectación de su función. Existe crecimiento de la aurícula derecha y habitualmente se asocia con comunicación interauricular (CIA), favoreciendo el shunt de derecha a izquierda. Las alteraciones anatómicas y hemodinámicas descritas condicionan el desarrollo de insuficiencia tricuspídea, arritmias supraventriculares y falla ventricular derecha'.

La AE tiene una incidencia de 1 cada 200.000 nacidos vivos y representa el 0,3 al 0,6\% de los pacientes con cardiopatías congénitas. Se asocia hasta en un 20-30\% con vías anómalas y síndrome de WolfParkinson-White (WPW)2.

Presentamos un caso clínico de un paciente con la coexistencia de AE y síndrome de WPW al que se le realizó estudio electrofisiológico con ablación exitosa de la vía anómala.

\footnotetext{
1. Residente de Cardiología.

2. Jefe de Residentes de Cardiología.

3. Médica de planta. Servicio de Electrofisiología y Arritmias.

4. Jefe de Servicio de Electrofisiología y Arritmias.

Instituto Cardiovascular de Rosario
}

$\triangle$ Correspondencia:Dra. María Florencia Firpo. mariaflorenciafirpo@gmail.com

\section{CASO CLÍNICO}

Paciente de sexo masculino de 38 años, con antecedentes familiares de cardiopatía isquémica (padre con infarto agudo de miocardio a los 47 años), con diagnóstico de AE a los 6 años y con el antecedente de ablación fallida de vía anómala hace 7 años en otra institución. Bajo tratamiento con flecainida 100 mg cada 12 horas. Presentó en los últimos 8 años episodios de palpitaciones paroxísticas, requiriendo internación por tal motivo en múltiples oportunidades, en una de las cuales se constató taquiarritmia regular con complejo QRS ancho con morfología de bloqueo completo de rama derecha, sin descompensación hemodinámica (Figura 1).

Fue evaluado en forma ambulatoria mediante electrocardiograma que evidencia signos de preexcitación ventricular (Figura 2), prueba ergométrica graduada, con desaparición del patrón de preexcitación durante el máximo esfuerzo, y ecocardiograma Doppler, que informó cavidades derechas severamente dilatadas e implantación baja de la valva septal de la válvula tricúspide compatible con AE.

Se decidió continuar la evaluación mediante estudio electrofisiológi$\mathrm{Co}$, en el cual se indujeron dos taquiarritmias: una taquicardia por reentrada auriculoventricular (TRAV) ortodrómica con conducción aberrante y una TRAV antidrómica (Figuras 3A y B). En base a dichos hallazgos se realizó ablación de vía anómala posteroseptal derecha, sin complicaciones (Figura 4)

Actualmente el paciente se encuentra asintomático, sin medicación antiarrítmica luego de dos años de seguimiento.

\section{DISCUSIÓN}

La expresión clínica de la AE es muy variable y depende fundamentalmente del grado de malformación anatómica del apara- 


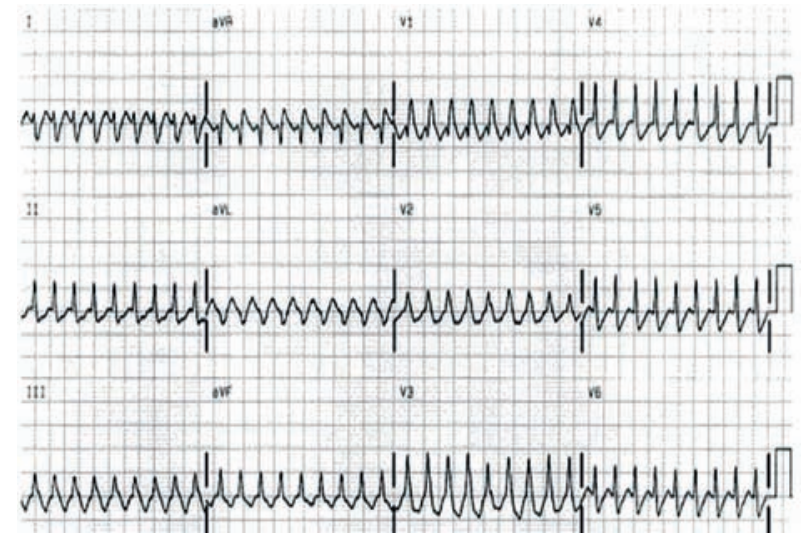

Figura 1. Taquiarritmia regular con complejo QRS ancho con morfología de bloqueo completo de rama derecha.

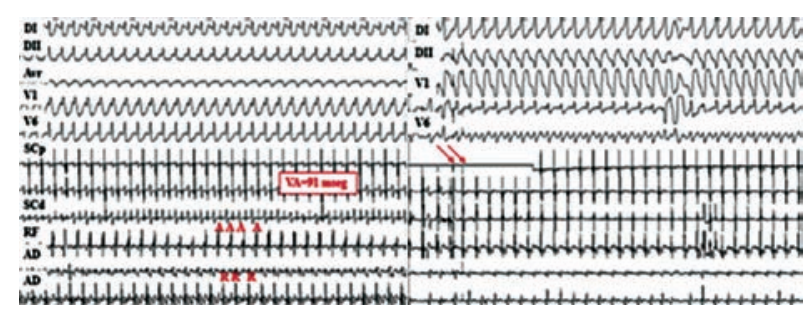

Figura 3. A) Taquicardia por reentrada auriculoventricular (AV) ortodrómica con conducción aberrante. B) Taquicardia por reentrada AV antidrómica con registros intracavitarios.

to valvular tricuspídeo, de la presencia de disfunción ventricular derecha y de la existencia de un cortocircuito derecha-izquierda'. Los síntomas como cianosis e insuficiencia cardíaca (IC) grave pueden aparecer a edades muy tempranas de la vida y con frecuencia mejoran al disminuir las resistencias vasculares pulmonares. En niños mayores de 10 años y en la edad adulta, la presencia de arritmias sintomáticas constituye la forma de presentación más frecuente ${ }^{3}$. Sin embargo, no es infrecuente que se manifieste con cianosis progresiva, disminución de la tolerancia al ejercicio, fatiga e IC derecha. En presencia de comunicación interauricular pueden sufrir embolias paradójicas, abscesos cerebrales y tienen mayor riesgo de muerte súbita.

Los pacientes con preexcitación presentan más frecuentemente fibrilación auricular (FA) y aleteo auricular (AA) que la población general ${ }^{4}$. Esto se debe, en parte, a la presencia de un período refractario auricular corto y una mayor vulnerabilidad auricular que favorece el desarrollo de arritmias supraventriculares. En caso de FA o AA, la presencia de una vía accesoria con conducción anterógrada puede desencadenar fibrilación ventricular y muerte súbita.

La ablación por radiofrecuencia en pacientes con cardiopatías congénitas es un desafío terapéutico debido a las alteraciones anatómicas descritas y a la frecuente asociación con múltiples vías anómalas. No obstante, es el tratamiento de elección en la actualidad para el manejo del síndrome de preexcitación ventricular, siendo un método seguro y efectivo en esta población de pacientes ${ }^{5}$. En varias muestras ob-

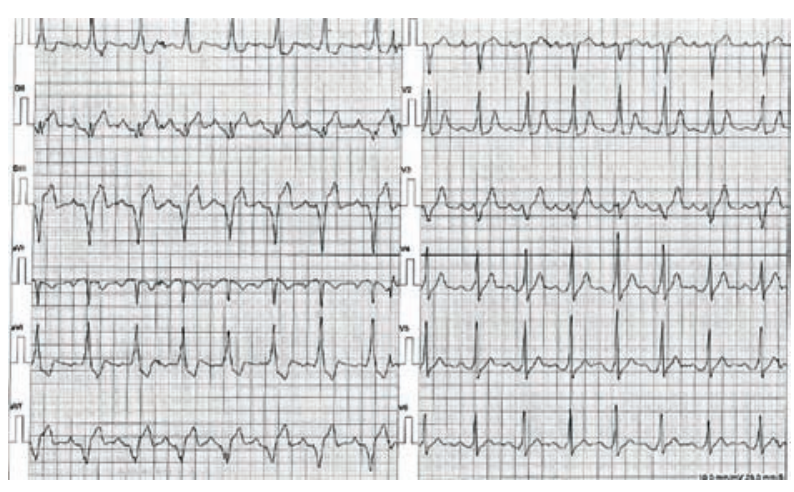

Figura 2. Electrocardiograma de ingreso. Ritmo sinusal con segmento PR corto y onda delta (flecha).

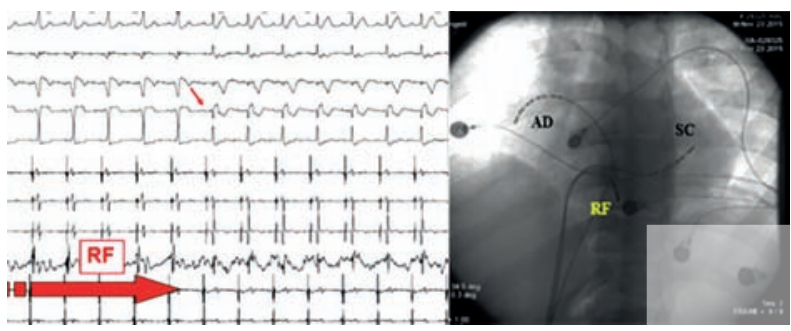

Figura 4. Electrocardiograma de superficie y registros endocavitarios donde se constata bloqueo de conducción por vía anómala durante aplicación de radiofrecuencia en el 60 latido (flecha). Fluoroscopia con el catéter de ablación posicionado en la región posteroseptal en el sitio de ablación exitosa. AD: aurícula derecha. SC: seno coronario. RF: catéter de radiofrecuencia.

servacionales de pacientes con WPW y AE se visualizó que la localización más frecuente de la vía anómala es la región posterior o posteroseptal (56\%), tal como lo encontrado en nuestro paciente 6 . La ablación por radiofrecuencia tiene una tasa de éxito del 90-95\%, siendo algo menor para las vías accesorias de localización septal

En un estudio descriptivo realizado por el Servicio de Electrofisiología y Arritmias de nuestra institución, con 178 pacientes (de febrero del 2003 a julio del 2016), con el objetivo de analizar la eficacia y seguridad de ablación por radiofrecuencia para el tratamiento de las arritmias en pacientes con vías anómalas, se demostró una elevada tasa de éxito inmediato $(92,13 \%)$ y un bajo porcentaje de complicaciones, de los cuales el $99 \%$ fueron menores.

\section{CONCLUSIÓN}

La AE es una malformación congénita infrecuente, asociada en un gran número de casos a síndromes de preexcitación. La incidencia de arritmias supraventriculares como FA es frecuente por la dilatación de cavidades derechas, y en pacientes con preexcitación puede significar alto riesgo de fibrilación ventricular. La ablación por radiofrecuencia tiene buenos resultados y es segura, por lo que representa el tratamiento de elección en síndrome de WPW, en especial en pacientes con anomalías congénitas. 


\section{BIBLIOGRAFÍA}

1. Attenhofer Jost C, Connolly HM, Dearani JA, Edwards WD, Danielson GK. Ebstein's Anomaly. Congenital Heart Disease for the Adult Cardiologist. Circulation 2007;115(2):277-285.

2. Iturralde Torres P. La anomalía de Ebstein asociada al sindrome de Wolff-Parkinson-White. Arch Cardiol Mex 2007;77(2):37-39.

3. Martínez-Losas P, Freitas-Ferraz A, Vivas D, Viana-Tejedor A. Síndrome de Wolff-Parkinson White como presentación de anomalia de Ebstein. Semergen 2015;41(6):52-54.

4. Iturralde P, Nava S, Sálica G, Medeiros A, Márquez MF, Colin L, et al. Electrocardiograph ic Characteristics of Patients with Ebstein's Anomaly Before and After Ablation of an ACcessory Atrioventricular Pathway. J Cardiovasc Electrophysiol 2006;17(12):1332-1336.
5. Kinoshita O, Agatsuma T, Hanaoka T, Tomita T, Tsutsui H, Yazaki Y, et al. Radiofrequency catheter ablation of accessory pathway in a patient with Ebstein's anomaly and atrial septal defect. A case report. Angiology 2005;56(2):221-223.

6. Betancourt Reyes G, Betancourt Betancourt G, González Pérez A. Síndrome de Wolff Parkinson White: reporte de un caso. Revista Cubana de Medicina Intensiva y Emergencias 2017;16(2):48-56.

7. Bartolomé F, Sánchez Fernández-Bernal C, Torres Feced F. Ablación con catéter de vías accesorias en pacientes con cardiopatías congénitas. Rev Esp Cardiol 1999;52(11):1028-1031 